\title{
Effect of Miraculin on Sweet and Sour Tastes Evoked by Mixed Acid Solutions
}

\author{
C. Endo, A. Hirata, A. Takami, I. Ashida*, Y. Miyaoka \\ Department of Health and Nutrition, Niigata University of Health and Welfare, Niigata, Japan \\ Email: ${ }^{*}$ asida@nuhw.ac.jp
}

Received 20 May 2015; accepted 8 June 2015; published 11 June 2015

Copyright (C) 2015 by authors and Scientific Research Publishing Inc.

This work is licensed under the Creative Commons Attribution International License (CC BY). http://creativecommons.org/licenses/by/4.0/

(c) (i) Open Access

\begin{abstract}
Miraculin is a glycoprotein, and it changes the perceived quality and intensity of tastes (especially, sourness and sweetness). The oral application of miraculin in a high concentration elicits a strong sweetness for sour tastants. However, the mixing of different tastants increases or decreases the intensity of the taste elicited in comparison with the intensities elicited by the individual sour tastants. The synergy between different (e.g., sweetness and sourness) or similar taste qualities can be responsible for the changes in the perceived taste intensities. Despite the characteristics of miraculin and the sour tastant mixtures, the effects of miraculin on mixed acid solutions are still unknown. The goals of this study were to assess the following: 1) the intensities of the sourness and sweetness of mixed acid solutions before and after the oral application of miraculin and 2) the intensity of the sweetness of the mixed acid solutions after the oral application of miraculin. For twenty healthy young adults, sensory evaluation experiments examined the perceived intensities of taste (sweetness, sourness, and astringency) and the overall odor of six organic acid solutions $\left(2.3 \times 10^{-2} \mathrm{M}\right.$ of citric acid and three-binary and two-trinary mixtures including citric acid that ranged from 2.24 to 2.48 in average $\mathrm{pH}$ ). The application of miraculin elicited sufficient sweetness, but it did not show statistically significant differences in the intensities of sweetness among the six acid solutions. Except for the sweetness, all of the four sensory items exhibited significant changes among the six solutions both before and after the application of miraculin, while the average scores for these items did not correlate with the average solution $\mathrm{pH}$ values. These results suggest that the binary and trinary mixed acid solutions affect the sourness both before and after the oral application of miraculan; however, there was no effect on the sweetness.
\end{abstract}

\section{Keywords}

Miraculin, Organic Acid Mixture, Sensory Evaluation, Young Adult

\footnotetext{
${ }^{*}$ Corresponding author.
}

How to cite this paper: Endo, C., Hirata, A., Takami, A., Ashida, I. and Miyaoka, Y. (2015) Effect of Miraculin on Sweet and Sour Tastes Evoked by Mixed Acid Solutions. Food and Nutrition Sciences, 6, 757-764. 


\section{Introduction}

Miraculin is a glycoprotein that changes sourness into sweetness in the mouth [1] [2]. When miraculin binds to the sweet receptors on the taste buds on the tongue or the soft palate, sour foods evoke an action potential on the taste nerves that conduct the sweet sensation. The intensity of sweetness becomes stronger as the intensity of sourness becomes stronger with organic acids (e.g., citric acid). The concentration of hydrogen ions affects the intensity of the sourness, although hydrogen ions are not the only determinant of taste [3].

When different tasting substances are mixed in solution and applied orally, their taste intensity is increased or decreased in comparison with the intensities elicited by the individual substances [4]; e.g., $0.5 \%$ sodium chloride $(\mathrm{NaCl})$ increases the sweetness of $5 \%-7 \%$ sucrose, while $1.0 \% \mathrm{NaCl}$ decreases the sweetness of $3 \%-10 \%$ sucrose [5]. A study on the mixing of solutions with similar taste intensities, specifically sour solutions, found that the sourness of binary acid mixtures is stronger than that of single acid solutions [6] [7].

Despite the fact that miraculin changes sourness to sweetness in single acid solution, few studies have examined the effect of miraculin on mixed acid solutions. The goals of the present study were to estimate 1) the intensity of the sourness of mixed acid solutions before oral miraculin was applied, and 2) the sweetness intensity of mixed acid solutions after miraculin was applied in response to the enhancement of the sourness.

\section{Materials and Methods}

\subsection{Participants}

Twenty adults (ten males and ten females, $20.7 \pm 0.8$ (mean \pm SD) years old) participated in this study. None of the participants had any subjective problems with gustatory and olfactory functions. Informed consent was obtained from all of the participants. The experiments were approved by the Ethics Committee of the Niigata University of Health and Welfare.

\subsection{Miraculin and Test Solutions}

A commercially available tablet of miraculin (MBerry [8]) was used in this study. The tablet was gently ground in a mortar and pestle. The ground powder $(350 \mathrm{mg})$ was provided to each participant with a plastic spoon. The efficacy of the ground miraculin powder was verified in a preliminary experiment (in six healthy young females).

Six acid solutions were used (Table 1); one single, three binary, and two trinary mixtures of four organic acids; citric acid (C) was a common component of the six solutions. The concentration of $\mathbf{C}$ was determined from the standard taste solution as in a previous report [9] and was approximately 10 times higher than the average recognition threshold of citric acid [10]. Table 1 shows the concentrations of the other three organic acids: malic acid (M), tartaric acid (T), and acetic acid (A). The concentrations of the three organic acids were determined based on the PSE (point of subjective equality) of the acid solutions [11]. The $\mathrm{pH}$ values of the six solutions used were measured at room temperature (approximately $25^{\circ} \mathrm{C}$ ) with a glass electrode $\mathrm{pH}$ meter (TWIN pH meter B-212, HORIBA Ltd., Kyoto, Japan). The average $\mathrm{pH}$ value ranged from 2.30 to 2.48 (Table 1). A one-way analysis of variance (ANOVA) detected significant differences among the average $\mathrm{pH}$ values $(P<0.01)$, and Tukey's multiple comparison tests followed by the ANOVA found nine specific differences in 15 possible pairs of solutions (Table $1, P \mathrm{~s}<0.001$ ).

Table 1. Average $\mathrm{pH}$ values of the used sour solutions and their statistical specific differences.

\begin{tabular}{|c|c|c|c|c|c|c|c|}
\hline Sour solution & $\mathrm{pH}$ & C & CM & CT & CA & CMA & CMT \\
\hline C & 2.48 & - & $* * *$ & $* * *$ & n.s. & $* * *$ & n.s. \\
\hline CM & 2.30 & - & - & n.s. & $* * *$ & n.s. & $* * *$ \\
\hline CT & 2.24 & - & - & - & $* * *$ & n.s. & $* * *$ \\
\hline CA & 2.48 & - & - & - & - & *** & n.s. \\
\hline CMA & 2.42 & - & - & - & - & - & $* * *$ \\
\hline CMT & 2.30 & - & - & - & - & - & - \\
\hline
\end{tabular}

Abbreviations are that: C, citric acid; M, malic acid; T, tartaric acid; and A, acetic acid. ${ }^{* * *} P<0.001$, n.s.: not significant. 


\title{
2.3. Procedures
}

The sensory evaluation experiment was conducted in an air-conditioned room at $25^{\circ} \mathrm{C}$. Each participant was seated in a chair in front of a table. Each participant was instructed to 1) place the miraculin powder in the mouth, and then 2) mix the powder with saliva and apply the saliva to the entire mucosae of the mouth with the tongue for $1 \mathrm{~min}$. After the application of the miraculin, the participant was instructed to rinse the mouth with 20 $\mathrm{ml}$ deionized water. The participant was asked to evaluate one of the test solution pairs (see the details in the next subsection) and rinse the mouth again with $20 \mathrm{ml}$ deionized water. An interval of 2 min was interposed between the individual trials.

\subsection{Sensory Evaluation}

A modification of Scheffe's paired comparison was used for the sensory evaluation. Each participant was provided a pair of test solutions $(15 \mathrm{ml})$ that were selected in random order. First, the participant tasted and smelled a solution and memorized the intensities of three qualities of the taste (sweetness, sourness, and bitterness) and the overall intensity of the odor. Second, after spitting out the previous solution with saliva and rinsing the mouth, the participant tasted and smelled the next solution and evaluated the taste in comparison to that of the previous solution. Finally, the participant spit out the solution with saliva and sufficiently rinsed the mouth. A trial consisted of these three steps. A conventional method was adopted for the evaluation [12]. The evaluation consisted of a grade composed of seven points (from -3 to +3 ). All of the possible 15 pairs of test solutions were used for the evaluation.

\subsection{Statistical Analysis}

The evaluated scores were analyzed statistically by a three-way ANOVA using "main effect (tastes or odor)", "participant", and "main × participant". After estimating the expected values of the main effect in the ANOVA, confidence intervals for the estimates were calculated using 'yardsticks' to detect specific differences between the pairs of solutions. Pearson's correlation coefficient was calculated between the sensory evaluation scores and $\mathrm{pH}$ values, i.e., the individual average scores of the seven evaluation items before (three items) and after (four items) miraculin was applied (Figure 1 and Figure 2) and the average $\mathrm{pH}$ values of the six acid solutions (Table 1). The statistical significance of the calculated coefficients was evaluated using a table of critical statistical values for Pearson's coefficient (e.g., Table B.7 in [13]). Differences were regarded as statistically significant if $P<0.05$.

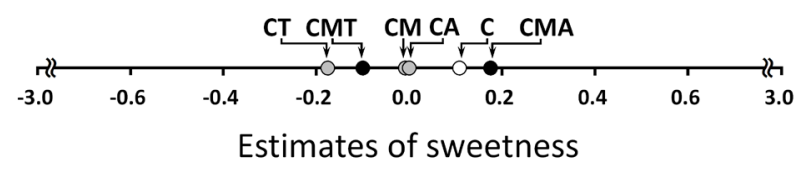

(a)



(b)

\begin{abstract}
Figure 1. Estimates of sweetness and sourness by sensory evaluation. (a) Intensity of sweetness in the six solutions (see Table 1) after miraculin was applied; (b) Intensities of sourness in the six solutions before (upper trace) and after (lower trace) miraculin was applied. White dot: a single solution; gray dots: three binary mixtures of organic acids; and black dots: two trinary mixtures of organic acids. See Table 2 and Table 3 for the specific statistical differences detected between pairs of solutions for sweetness (Table 2) and sourness (Table 3).
\end{abstract}




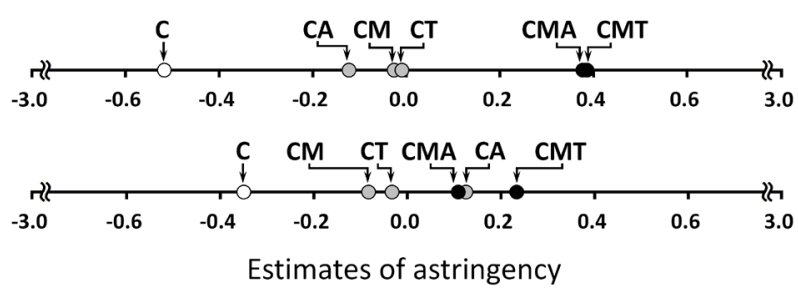

(a)

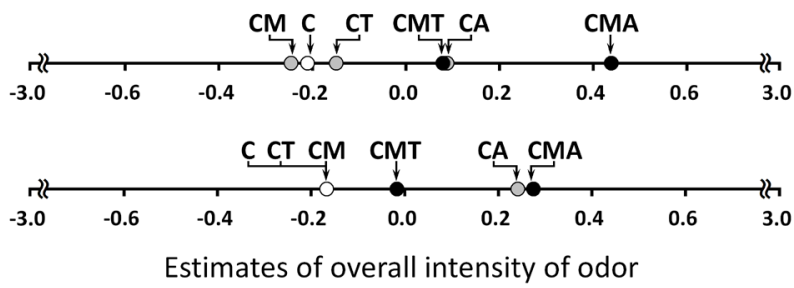

(b)

\begin{abstract}
Figure 2. Estimates of the astringency and overall odor intensity from the sensory evaluation. (a) Intensity of astringency in the six solutions (see Table 1) before (upper trace) and after (lower trace) miraculin was applied; (b) Intensity of overall intensity of odor in the six solutions before (upper trace) and after (lower trace) miraculin was applied. White dot: a single solution; gray dots: three binary mixtures of organic acids; and black dots: two trinary mixtures of organic acids. See Table 4 and Table 5 for the specific statistical differences detected between pairs of solutions for the astringency (Table 4) and the overall odor intensity (Table 5).
\end{abstract}

\title{
3. Results
}

Figure 1(a) shows estimates of the sweetness of six acid solutions after oral miraculin was applied. The highest estimate of +0.17 was elicited by the trinary mixture, CMA, while the lowest estimate of -0.17 was elicited by a binary mixture, CT. The range between these estimates, 0.34 , was only $5.7 \%$ of the possible parameter range (6.0; from -3.0 to +3.0), showing that the difference in the sweetness among the six solutions was relatively small. The three-way ANOVA did not detect significant differences in the "main effect (sweetness; $P=0.14$ )", or in "participant $(P=0.06)$ ", nor in the "main $\times$ participant interaction $(P=0.86)$ ", and the estimate of the confidence intervals between the solutions also indicated no specific differences (Table 2, $P s>0.05$ ).

Figure 1(b) shows the estimates of the sourness of six solutions before and after the oral miraculin was applied. The highest estimates were elicited by the trinary solution CMT (before miraculin; +0.58 ) and by the trinary solution CMA (after miraculin; +0.53), while the lowest estimates were elicited by a single solution of $\mathbf{C}$ both before and after the oral miraculin was applied ( -0.72 and -0.67 , respectively). The ranges of the estimates encompassed approximately $20 \%$ of the possible parameter range. The three-way ANOVA detected significant differences both before and after the miraculin application $(P s<0.01)$ (Table 3$)$ and specific differences were also found in 10 of the 15 (before miraculin) and 7 of the 15 (after miraculin) possible pairs of the acid solutions (shown by the lower and upper triangular elements in Table 4, respectively $(P<0.05)$.

Figure 2(a) and Figure 2(b) show the estimates of astringency and the overall odorintensity, respectively, for six acid solutions after the oral miraculin was applied. The highest and lowest estimates of the astringency were elicited by the trinary solution CMT and by the single solution of $\mathbf{C}$, respectively, before and after the miraculin was applied (Figure 2(a)). The highest estimates were +0.38 (before miraculin) and +0.23 (after miraculin), while the lowest estimates were -0.51 (before miraculin) and -0.35 (after miraculin). The three-way ANOVA detected significant differences both before and after the miraculin was applied $(P s<0.01)$, and specific differences were also found in 10 of 30 possible pairs of the acid solutions (Table 4, $P$ s $<0.05$ ). The highest overall intensity of odor was elicited by the two acid solutions, binary CA and trinary CMA, in which both contained A (Figure 2(b)). The three-way ANOVA detected significant differences both before and after miraculin was applied $(P s<0.01)$, and specific differences were also found in 12 of the 30 possible pairs of the acid solutions (Table 5, Ps $<0.05$ ).

Pearson's correlation coefficient indicated that the average $\mathrm{pH}$ values (Table 1) were not significantly corre- 
Table 2. Statistical specific differences on sweetness between pairs of solutions before and after application of miraculin.

\begin{tabular}{ccccccc}
\hline Sour solution & C & CM & CT & CA & CMA & CMT \\
\hline C & - & n.s. & n.s. & n.s. & n.s. & n.s. \\
CM & - & - & n.s. & n.s. & n.s. & n.s. \\
CT & - & - & - & n.s. & n.s. & n.s. \\
CA & - & - & - & - & n.s. & n.s. \\
CMA & - & - & - & - & - & n.s. \\
CMT & - & - & - & - & - & - \\
\hline
\end{tabular}

n.s.: not significant.

Table 3. Statistical specific differences on sourness between pairs of solutions before (under triangular) and after (upper triangular) application of miraculin.

\begin{tabular}{ccccccc}
\hline Sour solution & C & CM & CT & CA & CMA & CMT \\
\hline C & - & $*$ & $* *$ & $* *$ & n. & n.s. \\
CM & n.s. & - & n.s. & n.s. & n.t. \\
CT & $* *$ & n.s. & - & n.s. & $*$ & n.s. \\
CA & $* *$ & n.s. & n.s. & - & n.s. & n.s. \\
CMA & $* *$ & $* *$ & $*$ & $*$ & n.s. \\
CMT & $* *$ & $* *$ & $* *$ & & n.s. \\
\hline
\end{tabular}

${ }^{* *}: P<0.01 ;{ }^{*}: P<0.05 ;$ n.s.: not significant.

Table 4. Statistical specific differences on astringency between pairs of solutions before (under triangular) and after (upper triangular) application of miraculin.

\begin{tabular}{|c|c|c|c|c|c|c|}
\hline Sour solution & C & $\mathbf{C M}$ & CT & CA & СМА & CMT \\
\hline C & - & n.s. & n.s. & $* *$ & $* *$ & $* *$ \\
\hline CM & n.s. & - & n.s. & n.s. & n.s. & n.s. \\
\hline CT & * & n.s. & - & n.s. & n.s. & n.s. \\
\hline CA & n.s. & n.s. & n.s. & - & n.s. & n.s. \\
\hline CMA & $* *$ & * & n.s. & * & - & n.s. \\
\hline CMT & $* *$ & * & n.s. & * & n.s. & - \\
\hline
\end{tabular}

${ }^{* *}: P<0.01 ;{ }^{*}: P<0.05 ;$ n.s.: not significant.

Table 5. Statistical specific differences on overall intensity of odor between pairs of solutions before (under triangular) and after (upper triangular) application of miraculin.

\begin{tabular}{|c|c|c|c|c|c|c|}
\hline Sour solution & C & $\mathbf{C M}$ & CT & CA & CMA & CMT \\
\hline $\mathrm{C}$ & - & n.s. & n.s. & ** & ** & n.s. \\
\hline $\mathbf{C M}$ & n.s. & - & n.s. & ** & ** & n.s. \\
\hline CT & n.s. & n.s. & - & ** & $* *$ & n.s. \\
\hline CA & n.s. & * & n.s. & - & n.s. & n.s. \\
\hline CMA & $* *$ & ** & ** & * & - & n.s. \\
\hline CMT & n.s. & n.s. & n.s. & n.s. & * & - \\
\hline
\end{tabular}

${ }^{* *}: P<0.01 ;{ }^{*}: P<0.05 ;$ n.s.: not significant. 
lated with any of the average scores in the four sensory evaluation items (Figure 1 and Figure 2) both before (sourness, $r=-0.301$; astringency, -0.431 ; and overall odor, 0.296 ) or after miraculin application (sweetness, 0.766; sourness, -0.076 ; astringency, -0.240 ; and overall odor, 0.524 ).

\section{Discussion}

The most important result of this study (Figure 1(a)) indicated that miraculin did not change the intensity of the sweetness of the six acid solutions used (Table 1). Prior to discussing this result, it is necessary to discuss the taste intensities (specifically the sourness) before the oral miraculin was applied, because our hypothesis about the sweetness was based on the changes in the sourness of the acid solutions.

1) Before miraculin application

The concentration of hydrogen ions in a solution (i.e., $\mathrm{pH}$ ) critically affects the sourness intensity, although the hydrogen ion is not the sole determinant of taste [14]. This study found significant differences in the average $\mathrm{pH}$ values among the six solutions used (Table 1), and these differences did not determine the intensities of the sensory evaluation items including sourness before and after miraculin was applied, as the average $\mathrm{pH}$ values were not significantly correlated with any of the sensory intensities. The result of the perceived sourness intensity (Figure 1(b)) might provide additional information about the knowledge of the taste elicited by mixtures of acid solutions. This knowledge most likely originates from two major studies [7] [15]; the latter one used suprathresold acid solutions similar to those used in the present study, and that finding is likely to be more informative than that of the former study. A psychophysical study showed that estimates of sourness for binary mixtures and citric acid and $\mathrm{HCl}$ mixtures served as examples of "synergy" [7], although the other mixtures in that study differ from those in the present study (Table 1). Many studies have examined the different tastes of combinations, such as sweetness and sourness, but many fewer studies have done so with the same or similar qualities. The present result (Figure 1(b)) may extend 'synergy' in sourness to binary and trinary mixtures in acid solutions.

\section{2) After miraculin application}

The major finding regarding the sweetness was quite unexpected, as previous studies showed the enhancement of sourness with binary acid solutions [7] [15]; accordingly, we expected the enhancement of sweetness with binary acid solutions after the oral miraculin was applied. In fact, differential intensities of sourness in binary and trinary organic acid solutions were indeed observed in the present study (Figure 1(b)). A psychophysical study showed that the perceived intensity of sweetness after the miraculin was applied increased with an increase in the concentration of an acid solution (citric acid, $8.5 \times 10^{-4}$ to $1.38 \times 10^{-2} \mathrm{M}$; and hydrochloric acid, $7.8 \times 10^{-4}$ to $1.29 \times 10^{-2} \mathrm{M}$ ) that was tasted after the miraculin [16]. An electrophysiological study in monkeys also showed that the response of the summed activity of the chorda tympani nerve to a citric acid solution was greater after miraculin application, when the concentrations were increased from $1.0 \times 10^{-2} \mathrm{M}$ to $5.0 \times 10^{-2} \mathrm{M}$ [17]. These studies in humans and monkeys suggest that stronger sourness from higher concentrations of acid solutions elicits stronger sweetness. However, a psychophysical study in humans also suggests that the miraculin effect reaches a peak at a concentration of $10^{-2} \mathrm{M}$ citric acid (Figure 1 in [16]). This peak may be responsible for the negative result in the present study (Figure 1(a)), as the concentration of citric acid used (Table 1) was similar to the highest concentration used in a previous study [16]. The peak effect of miraculin is consistent with solutions of $\mathrm{HCl}$ (Figure 2 in [16]), but no previous studies have explored the effects, including a peak, of miraculin on binary or trinary acid solutions. The negative result suggests that the peak effect of miraculin occurs with binary and trinary acid solutions such as those used in the present study. Despite this indication, a question remains, because while the average $\mathrm{pH}$ values differed significantly among the test solutions (Table 1), there were no differences in the sweetness intensity after miraculin was applied. The peak effect is unlikely to be a sufficient answer to this question. It seems necessary to consider other possibilities such as astringency and the overall odor intensity of the tested substances for the answer.

In our preceding paper [9], the participants estimated the perceived intensity of sweetness as an average score of approximately 133, which was elicited by a solution of $2.3 \times 10^{-2} \mathrm{M}$ citric acid after miraculin was applied, while the participants were instructed to consider the sweetness elicited by a solution of $0.20 \mathrm{M}$ sucrose before miraculin was applied as 100 (i.e., the standard score). That study used the citric acid solution and five other acid solutions, and a wine vinegar containing tartaric and acetic acids was one of the five solutions [9]. The wine vinegar showed the lowest average score for the sweetness of the five solutions after miraculin was applied, 
while the $\mathrm{pH}$ measured was at the lowest value. A previous study first showed that astringency elicited by acids is a function of $\mathrm{pH}$ and not of the concentration or of the anion species [18]. A more recent study also showed that an increase in $\mathrm{pH}$ from 2.5 to 4.0 in wines lowered the astringency intensity [19]. Thus, the present result that indicated that the two trinary acid solutions showed higher astringency scores than the remaining four solutions is consistent with these previous studies. That the wine vinegar had the lowest $\mathrm{pH}$ [9] suggests that it should have the highest astringency of the six solutions tasted [18] [19]. Findings in previous studies, including one study of ours, suggest that the astringency of the solution could have been a factor in the unexpected negative result found in this study (Figure 1). However, it is still unclear why the appliedmiraculin did not differentially modify the sweetness of the six acid solutions used in this study (Figure 1(a) and Table 2).

The overall odor intensity of the acid solutions (Figure 2(b)) can affect the perceived intensity of sweetness after miraculin application (Figure 1(a)). The acetic acid content of the solutions seems to be responsible for the high overall odor intensity scores both before and after miraculin application (Figure 2(b) and Table 5). In general, tastes and smells usually interact in a suppressive manner at suprathreshold concentrations [20]-[22], although the methods used for sensory evaluation are likely to be critical [23]. The average sweetness intensity in three acid solutions that included acetic acid after miraculin application tended to be lower than in those solutions without acetic acid in our preceding study [9]. The present result for the overall odor intensity suggests a minor influence on the sweetness intensity of the mixed acid solutions after the miraculin application.

\section{Conclusion}

Effects of ground miraculin powder on the perceived intensity of taste and odor were evaluated using six (one single, three binary, and two trinary) organic acid solutions in twenty healthy young adults. Citric acid ( $2.3 \times$ $10^{-2} \mathrm{M}$ ) was a component of the solutions, and the average $\mathrm{pH}$ values were between 2.24 and 2.48 . The ground miraculin powder elicited sufficient sweetness after oral application. In contrast to our prediction, no statistically significant differences were found in the intensities of sweetness elicited by the miraculin application among the six acid solutions. Significant differences after miraculin was applied were found in three of the sensory items evaluated (the sourness, the astringency, and the overall odor intensity) among the six solutions, while the average scores for these items were not correlated with the average solution $\mathrm{pH}$ values. These results suggest that the sweetness elicited by miraculin is not enhanced or at least synergized by the binary and trinary acid solutions used in this study.

\section{References}

[1] Cagan, R.H. (1973) Chemostimulatory Protein: A New Type of Taste Stimulus. Science, 181, 32-35. http://dx.doi.org/10.1126/science.181.4094.32

[2] Theerasilp, S., Hitotsuya, H., Nakajo, S., Nakaya, K., Nakamura, Y. and Kurihara, Y. (1989) Complete Amino Acid Sequence and Structure Characterization of the Taste-Modifying Protein, Miraculin. Journal of Biological Chemistry, 264, 6655-6659.

[3] Ganzevles, P.G. and Kroeze, J.H. (1987) Effects of Adaptation and Cross-Adaptation to Common Ions on Sourness Intensity. Physiology \& Behavior, 40, 641-646. http://dx.doi.org/10.1016/0031-9384(87)90111-9

[4] Pangborn, R.M. (1960) Taste Interrelationships. Food Science, 25, 245-256. http://dx.doi.org/10.1111/j.1365-2621.1960.tb00328.x

[5] Sjöström, L.B. and Cairncross, S.E. (1953) Role of Sweeteners in Food Flavor. Advances in Chemistry Series, 108113.

[6] Bartoshuk, L.M. and Cleveland, C.T. (1977) Mixtures of Substances with Similar Tastes. A Test of a Psychophysical Model of Taste Mixture Interactions. Sens Processes, 1, 177-186.

[7] Moskowitz, H.R. (1974) Sourness of Acid Mixtures. Journal of Experimental Psychology, 102, 640-647. http://dx.doi.org/10.1037/h0036082

[8] MBerry: Makes Life Sweeter. http://mberry.us/

[9] Igarashi, G., Higuchi, R., Yamazaki, T., Ito, N., Ashida, I. and Miyaoka, Y. (2013) Differential Sweetness of Commercial Sour Liquids Elicited by Miracle Fruit in Healthy Young Adults. Food Science and Technology International, 19, 243-249.

[10] Pfaffmann, C., Bartoshuk, L.M. and Mcburney, D.H. (1971) Taste Psycophysics. In: Beidler, L.M., Ed., Handbook of Sensory Physiologt, Springer-Verlag, Berlin/Heidelberg/New York, 75-101. 
[11] Pangborn, R.M. (1963) Relative Taste Intensities of Selected Sugars and Organic Acids. Food Science, 28, 726-733. http://dx.doi.org/10.1111/j.1365-2621.1963.tb01680.x

[12] Lawless, H.T. and Heymann, H. (1999) Discrimination Testing. In: Lawless, H.T., Ed., Sensory Evaluation of Food, Aspen Publication, Gaithersburg, 116-139. http://dx.doi.org/10.1007/978-1-4419-7452-5_4

[13] Weathington, B.L., Cunningham, C.J.L. and Pittenger, D.J. (2012) Appendix B: Statistical Tables. In: Weathington, B.L., Cunningham, C.J.L. and Pittenger, D.J., Eds., Understanding Business Research, Wiley \& Sons. Inc., Canada, 435-483. http://dx.doi.org/10.1002/9781118342978.app2

[14] Richards, T.W. (1900) The Relation of the Taste of Acids to Their Degree of Dissociation, II. Journal of Physical Chemistry, 4, 207-211. http://dx.doi.org/10.1021/j150021a005

[15] Hahn, H. and Ulbrich, L. (1948) Eine systematische Untersuchung der Geschmacksschwellen. Pflugers Arch Gesamte Pflügers Archiv für die Gesamte Physiologie des Menschen und der Tiere, 250, 357-384. http://dx.doi.org/10.1007/BF00369741

[16] Bartoshuk, L.M., Gentile, R.L., Molkowitz, H.R. and Meiselman, H.L. (1974) Sweet Taste Induced by Miracle Fruit (Synsepalum dulcificum). Physiology \& Behavior, 12, 449-456. http://dx.doi.org/10.1016/0031-9384(74)90122-X

[17] Hellekant, G., Hagstrom, E.C., Kasahara, Y. and Zotterman, Y. (1974) On the Gustatory Effects of Miraculin and Gymnemic Acid in the Monkey. Chemical Senses and Flavor, 1, 137-145. http://dx.doi.org/10.1093/chemse/1.2.137

[18] Sowalsky, R.A. and Noble, A.C. (1998) Comparison of the Effects of Concentration, pH and Anion Species on Astringency and Sourness of Organic Acids. Chemical Senses, 23, 343-349. http://dx.doi.org/10.1093/chemse/23.3.343

[19] Fontoin, H., Saucier, C., Teissedre, P.L. and Glories, Y. (2008) Effect of pH, Ethanol and Acidity on Astringency and Bitterness of Grape Seed Tannin Oligomers in Model Wine Solution. Food Quality and Preference, 19, $286-291$. http://dx.doi.org/10.1016/j.foodqual.2007.08.004

[20] Garcia-Medina, M.R. (1981) Flavor-Odor Taste Interactions in Solutions of Acetic Acid and Coffee. Chemical Senses, 6, 13-22. http://dx.doi.org/10.1093/chemse/6.1.13

[21] Gillan, D.J. (1983) Taste-Taste, Odor-Odor, and Taste-Odor Mixtures: Greater Suppression within than between Modalities. Perception \& Psychophysics, 33, 183-185. http://dx.doi.org/10.3758/BF03202837

[22] Breslin, P.A.S. (2000) Human Gustation. In: Finger, T.E., Silver, W.L. and Restrepo, D., Eds., The Neurobiology of Taste and Smell, Wiley-Liss, Inc., New York, 423-461.

[23] Lawless, H. and Schlegel, M.P. (1984) Direct and Indirect Scaling of Sensory Differences in Simple Taste and Odor Mixtures. Journal of Food Science, 49, 44-46. http://dx.doi.org/10.1111/j.1365-2621.1984.tb13665.x 